\title{
mTOR as a multifunctional therapeutic target in HIV infection
}

\section{Ferdinando Nicoletti ${ }^{1}$, Paolo Fagone ${ }^{1}$, PierLuigi Meroni ${ }^{2}$, James McCubrey ${ }^{3}$ and Klaus Bendtzen ${ }^{4}$}

\footnotetext{
${ }^{1}$ Department of Bio-Medical Sciences, School of Medicine, University of Catania, Italy

${ }^{2}$ Istituto Auxologico, University of Milan, Italy

${ }^{3}$ Department of Microbiology and Immunology, Brody School of Medicine at East Carolina University, Greenville, NC, USA

${ }^{4}$ Institute for Inflammation Research (IIR), Rigshospitalet University Hospital, Copenhagen, Denmark
}

Patients undergoing long-term highly active antiretroviral therapy treatment are probably at a higher risk of various HIV-related complications. Hyperactivation of The mammalian target of rapamycin (mTOR) has been found to contribute to dysregulated apoptosis and autophagy which determine CD4 ${ }^{+}$-T-cell loss, impaired function of innate immunity and development of neurocognitive disorders. Dysregulated mTOR activation has also been shown to play a key part in the development of nephropathy and in the pathogenesis of HIV-associated malignancies. These studies strongly support a multifunctional key role for mTOR in the pathogenesis of HIV-related disorders and suggest that specific mTOR inhibitors could represent a novel approach for the prevention and treatment of these pathologies.

mTOR and its pharmacological inhibitors: multifunctional agonists and antagonists regulating immune functions, cancer, aging, viral infection and autism spectrum disorders

The mammalian target of rapamycin (mTOR), also known as mechanistic target of rapamycin or FK506-binding protein 12rapamycin-associated protein 1 (FRAP1), is a $\sim 289 \mathrm{kDa}$ protein originally discovered and cloned from Saccharomyces cerevisiae that shares sequence homologies with the phosphoinositide 3-kinase (PI3-kinase) family and represents a serine/threonine protein kinase that is present in all eukaryotic organisms [1].

mTOR plays a key part as an intracellular nutrient sensor that controls protein synthesis, cell growth and metabolism [2]; it is activated by different stimuli such as amino acids, growth factors and oxygen. mTOR represents the catalytic subunit of two distinct complexes, called mTORC1 and mTORC2 [2]. mTORC1 controls cell growth by maintaining a balance between anabolic processes, such as macromolecular synthesis and nutrient storage, and catabolic processes, such as autophagy and the utilization of energy stores $[3,4]$. mTORC2 regulates cell survival and cytoskeletal orga- nization through the regulation of Akt and protein kinase $\mathrm{C}$ alpha $(\mathrm{PKC} \alpha)$, respectively [5].

Rapamycin (Rapamune ${ }^{\circledR}$; sirolimus) is a macrolide first isolated from the soil bacterium Streptomyces hygroscopicus [6]. It represents the first-in-class of specific mTOR inhibitors. This class also includes derivatives, termed rapalogs, which include temsirolimus, everolimus and ridaforolimus. Some of these drugs have been approved for the prevention of graft rejection and the treatment of cancers. However, in agreement with the pleiotropic role of mTOR in cell biology, several experimental studies indicate beneficial effects of mTOR inhibitors in other conditions that range from Parkinson's disease to aging, viral infection and autism [7-9].

When rapamycin binds to its intracellular receptor FK-binding protein 12 (FKBP12) a complex is formed (rapamycin-FKBP12), and this inhibits the ability of mTOR to phosphorylate the p70 ribosomal protein S6 kinase 1 (p70S6K) [10]. In doing so, rapamycin interferes with the PI3K/Akt/mTOR axis which plays a key part in several cellular functions, including differentiation, viability and growth $[2,11]$. When the PI3K pathway is activated (e.g. by stress or growth factors), the consequent phosphorylation of Akt activates TSC2, a large protein that, together with TSC1 
(Tuberous Sclerosis 1), forms the TSC1-TSC2 complex. TSC1TSC 2 acts as a GTPase-activating protein (GAP) for Ras homologue enriched in brain (RHEB). GDP-bound RHEB cannot activate mTORC1, thus turning off the mTORC1 signalling pathway. Akt-mediated TSC2 phosphorylation is thought to inhibit the GAP activity for RHEB, thus promoting mTORC1 activation. By inhibiting mTOR, rapamycin blocks the above-mentioned signalling cascade downstream; thus modulating key events controlling cellular growth [2]. A diagram depicting the PI3K/PTEN/Akt/ mTOR pathway where rapamycin, rapalogs, PI3K/mTOR and mTOR inhibitors exert their effects on protein translation is presented in Fig. 1.

Rapamycin also possesses in vitro and in vivo anti-HIV properties that qualify it as a potential new anti-HIV drug [12]. Indeed, rapamycin represses HIV-1 replication in vitro through different mechanisms including, but not limited to, downregulation of C-C chemokine receptor type 5 (CCR5) (reviewed in Ref. [12]). In addition, rapamycin synergistically enhances the anti-HIV activity of entry inhibitors such as vicriviroc, aplaviroc and enfuvirtide in vitro [12]. Rapamycin also inhibits HIV-1 infection in human peripheral blood leukocyte reconstituted SCID mice [13], and a recent prospective trial of liver-transplanted HIV patients indicated significantly better control of HIV and hepatitis $\mathrm{C}$ virus replication in those receiving rapamycin monotherapy [14].

\section{HIV and its treatment: advantages and some limits of HAART}

HIV is a genetically related member of the Lentivirus genus of the Retroviridae family that shows a particular tropism for $\mathrm{CD} 4^{+} \mathrm{T}$ cells [15]. However, the CD4 antigen alone is not sufficient for virus entry, because a co-receptor is necessary to gain access into the cells. These molecules belong to the family of seven-transmembrane domain G-protein-coupled receptors. Typically, CXCR4 is used by strains with tropism for T cells and CCR5 by strains with tropism for macrophages [16].

The course of HIV infection is characterized by a phase of acute viremia associated with a rapid decrease in circulating $\mathrm{CD} 4^{+} \mathrm{T}$ cells [17]. It has been shown that, despite a massive immune response, the host fails to eliminate the virus which remains latent in the body [18]. This condition allows the virus to establish a chronic phase with progressive immunodeficiency that is determined primarily by high cell turnover, immune activation and apoptosis of $\mathrm{CD}^{+} \mathrm{T}$ cells [19].

Highly active antiretroviral treatment (HAART) consists of a combination of different drugs that inhibit HIV replication [20]. Although HAART accounts for the significant decrease in AIDSrelated morbidity and mortality seen in recent years [21], an increased rate of AIDS-related complications such as neurocognitive disorders, nephropathy and malignancies are being observed in patients with poor initial $\mathrm{CD} 4^{+}$recovery and in patients with a CD4 ${ }^{+}$count higher than 200 cells per $\mathrm{mm}^{3}$ $[22,23]$. This could be caused by prolonged viral persistence, chronic immune activation, incomplete immune reconstitution and/or accelerated immune senescence. Thus, although HAART has significantly increased the life expectancy of HIV-seropositive patients, several issues remain and new and/or adjuvant therapies are therefore strongly justified.

\section{Can mTOR dysregulation link HIV-1 infection to CD4 ${ }^{+}-\mathrm{T}^{-}$ cell loss, impaired function of innate immunity, dementia, nephropathy and malignancies?} mTOR and $C D 4^{+}-T$-cell loss

mTOR activation seems to play a pathogenetic part in the dysregulated apoptosis of $\mathrm{CD}^{+}{ }^{+} \mathrm{T}$ cells in HIV-1 patients through gp120mediated activation of the CD4/CXCR4 (or CCR5)/mTOR/p53 axis [24]. In fact, mTOR can phosphorylate p53 on serine 15 involved in Env-induced syncytial apoptosis [25]. The complex of the Env glycoprotein expressed on the membrane of HIV-1-infected cells can also induce apoptosis of uninfected cells expressing CD4 and CXCR4 or CCR5 through interaction between Env-expressing infected cells and uninfected cells [24,25].

It has been proposed that autophagy also plays a pathogenetic part in the death of uninfected $\mathrm{CD}^{+} \mathrm{T}$ cells [26]. Because mTOR inhibits the cellular mechanism of autophagy, this makes the net contribution of mTOR to the mechanism of CD4 ${ }^{+}$-T-cell depletion more complex to decipher. However, a study questioned the contribution of autophagy to $\mathrm{CD} 4^{+}$-T-cell death [27] because the authors reported reduction of the autophagy proteins Beclin-1 and LC3-II following infection of human peripheral blood $\mathrm{CD}^{+}{ }^{+} \mathrm{T}$ cells or U937 cells with HIV-1. Beclin-1 mRNA expression and autophagosomes were also reduced in HIV-1-infected cells $[27,28]$. The in vivo evidence indicating that the blockade of mTOR with rapamycin neither prevents $\mathrm{CD} 4^{+}$-T-cell decline in HIV-1infected SCID mice [13] nor fails to influence their numbers in $\mathrm{HIV}$-infected individuals receiving liver transplantation [14] seems to suggest that promotion of autophagy via mTOR blockade does not influence HIV-1-induced $\mathrm{CD} 4^{+}$-T-cell death in vivo.

\section{mTOR and impaired function of innate immunity during HIV infection}

Defective immunological function of cells of the macrophage lineage is known to contribute to the pathogenesis of HIV-1 infection. Thus, stopping phagocytosis of opportunistic pathogens such as Mycobacterium avium complex, Pneumocystis carinii, Toxoplasma gondii or Candida albicans by peripheral blood monocytes, tissue macrophages and monocyte-derived macrophages following in vivo and in vitro HIV-1 infection has been documented [29]. Late-stage development of opportunistic infections has therefore been attributed to defective monocyte/macrophage signalling and function in HIV-infected individuals [30].

mTOR activation inhibits the destruction of pathogenic microorganisms by macrophages by inhibiting autophagy; rapamycin restores this macrophage inhibition. Cells infected with HIV-1 blocked rapamycin-induced autophagy and CD40-induced autophagocytosis of T. gondii in bystander macrophage/monocytic cells. These studies show that a pathogenic microorganism can impair autophagy in non-infected cells by activating counter-regulatory pathways, and that activation of mTOR is involved in this process. In agreement with these findings it has also been demonstrated in dendritic cells (DCs) that rapamycin-mediated autophagy induction exerts a better anti-BCG vaccine response [31]. This raises the possibility of therapeutic manipulation of cell signalling to restore autophagy in HIV-1 infection [30].

A recent study also shows that HIV-1 can impair innate immune function by mTOR-mediated impaired autophagy of DCs [32]. The limited immune response seen in the early events of HIV-1 trans- 


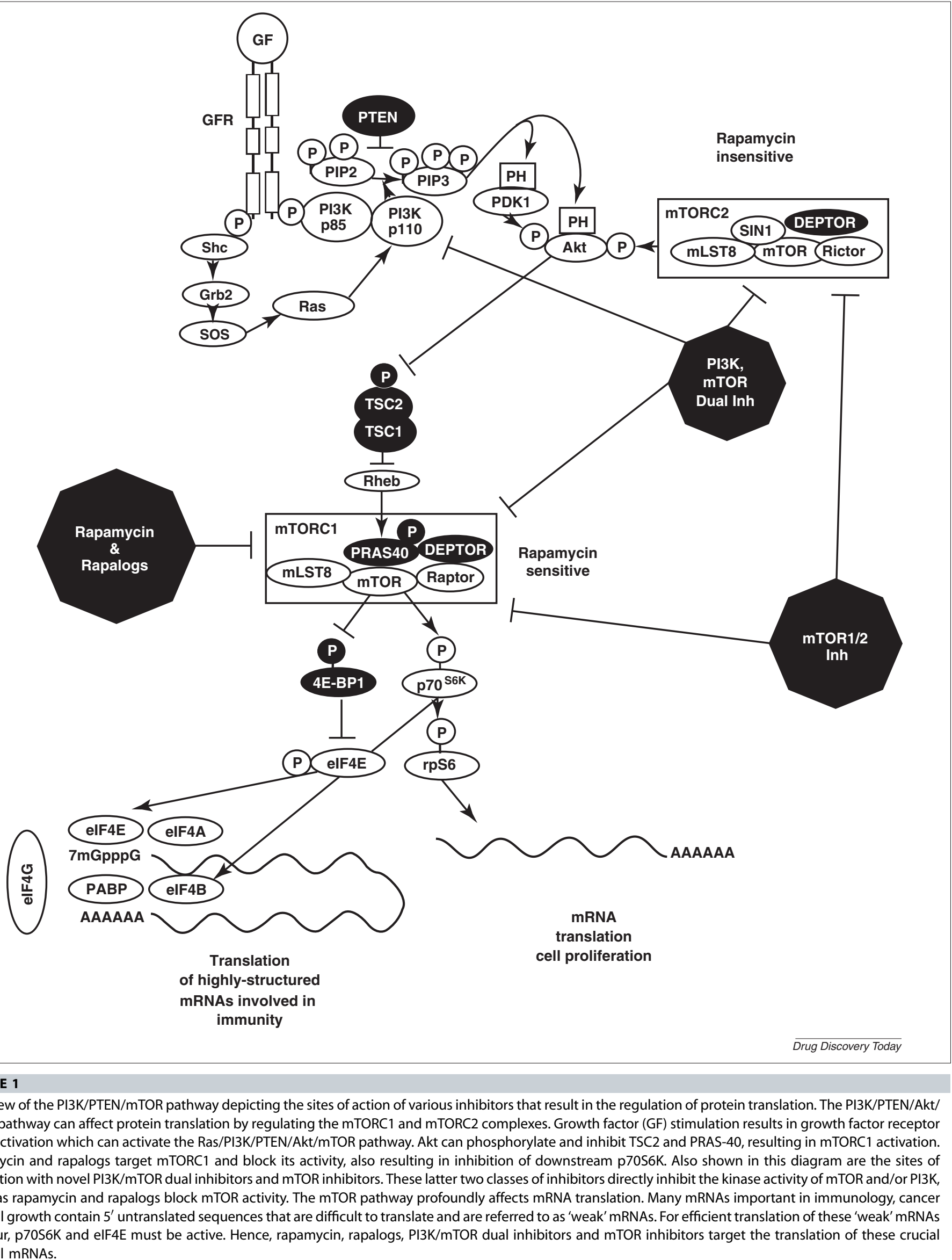


mission could result from the shutdown of autophagy and immunoamphisomes in DCs that rely on mTOR-dependent pathways [32]. HIV-1-induced inhibition of autophagy in DCs increases cellassociated HIV-1 and transfer of HIV-1 infection to $\mathrm{CD} 4^{+} \mathrm{T}$ cells. HIV-1- and mTOR-mediated downregulation of autophagy in DCs impaired innate and adaptive immune responses. Immunoamphisomes in DCs engulf incoming opportunistic infections and amplify pathogen degradation as well as Toll-like receptor responses and antigen presentation [32]. The findings that HIV1 downregulates autophagy and impedes immune functions of DCs in an mTOR-dependent manner could have therapeutic and prophylactic implications for the use of specific mTOR inhibitors.

\section{mTOR and HIV-associated neurocognitive disorders}

With the introduction of HAART, AIDS dementia complex - or HIV-associated dementia as it was termed later - largely disappeared from clinical practice. However, during the past few years long-term infected and treated patients, including those with wellcontrolled infection, started to complain about milder memory problems such as slowness and difficulties in concentration, planning and multitasking. Neuropsychological studies have confirmed that cognitive impairment occurs in $15-50 \%$ of patients, being one of the most feared complications of HIV-1-infection. Neurocognitive impairment can also affect adherence to treatment and, ultimately, result in increased morbidity $[33,34]$.

HIV-related neuropathology consists of microglial infiltration throughout white and grey matter, reactive astrogliosis in the cortex and in the central grey structures, and loss of neurons in the hippocampus and in the basal ganglia [35]. HIV enters the CNS through infected macrophages and resides in the microglia. The number of infected glial cells in HIV patients is highly variable but generally too low to explain the underlying process of encephalitis $[33,34]$. Thus, it is believed that immunoinflammatory and/or degenerative responses of the microglial cells to infection rather than virus-mediated cell death can explain AIDS-related neuropathogenesis $[33,34]$.

Alizarei and co-workers have recently studied the role of autophagy in microglia-induced neurotoxicity in primary rodent neurons, as well as in primate and humans models $[36,37]$. They found that products of microglia infected with simian immunodeficiency virus (SIV) inhibited neuronal autophagy, resulting in decreased neuronal survival. This was associated with a decrease in autophagyinducing proteins, a decrease in neuronal autophagy vesicles and an increase in sequestosome-1/p62. Signs of autophagy dysregulation associated with dementia and encephalitis were also found in brains from HIV-infected individuals and SIV-infected monkeys. Assessment of multiple biochemical markers of autophagic activity confirmed the inhibition of autophagy in neurons.

The contribution of dysregulated mTOR activity to this process was confirmed by the induction of autophagy in neurons after rapamycin treatment, and such treatment conferred significant protection to neurons $[36,37]$. This complements a previous study by Narducci and co-workers that showed that upregulation of mTOR and PUMA a p53 inducible BH3-only protein, also occurs in neurons of patients suffering from HIV-associated encephalitis [38]. Taken together, these results prove that defects in autophagy are involved in neurodegenerative processes that arise from glial, as opposed to neuronal, sources, and that maintenance of autop- hagy could have a role in neuroprotection during HIV infection. The decreased neuronal autophagy might sensitize cells to proapoptotic and other damaging mechanisms, leading to neuronal dysfunction and death. Hence, new therapeutic approaches aimed at boosting neuronal autophagy including mTOR blockade with rapamycin are conceivable to prevent the development of HIVassociated neurocognitive disorders and to treat those suffering from these complications.

\section{mTOR and HIV-associated nephropathy}

HIV-associated nephropathy (HIVAN) is an important clinical manifestation of HIV infection that develops in $~ 33 \%$ of HIV-1 cases [39]. Clinically evident chronic kidney disease and subclinical renal pathology can occur in patients with long-lasting HIV infection. The pathogenesis of HIVAN involves the direct infection of the renal epithelium following HIV infection [40].

The introduction of combination HAART has been followed by a decline in the incidence of HIVAN [41] and with delayed progression of end-stage renal disease.

The fact that mTOR could be involved in the pathogenesis of HIVAN is suggested by a recent study carried out in Tg26 transgenic mice that harbour the proviral transgene pNL4-3:d1443, which encodes all of the HIV-1 genes with the exception of GAG and $P O L$ and serves as a preclinical model of HIVAN [42]. These animals develop proteinuria at $\sim 24$ days of age and progress into nephritic syndrome and renal failure. Parietal and visceral epithelial cells had increased levels of mTOR phosphorylation when compared with the $\mathrm{FVB} / \mathrm{N}$ control mouse [42], and treatment with rapamycin reduced the renal lesions, proteinuria and uremia. Rapamycin also decreased tubular dilation, probably caused by inhibition of protein synthesis and cell cycle progression in tubular cells [42]. These findings concur with a role for mTOR in the proliferative phenotype and the development of HIVAN, and provide in vivo proof-of-concept for the beneficial effects of mTOR inhibitors in this setting

\section{mTOR and HIV-associated malignancies}

The occurrence of HIV-associated malignancies represents a major complication for immunocompromised HIV-infected individuals [43]. Indeed, it has been shown that there is an association between HIV and cancer, particularly anal cancer, Kaposi sarcoma (KS), non-Hodgkin's lymphoma (NHL), primary effusion lymphoma (PEL), primary brain lymphoma and Burkitt's lymphoma (BL) [44]. Several factors have been identified as culprits of malignancies in the HIV patients, including impaired immunocompetence, dysregulated immune responses, genomic instability, chronic B-cell stimulation and opportunistic infections with oncogenic viruses [45]. The severity of immune suppression is predictive of mortality from AIDS- and non-AIDS-associated malignancies and it has been proposed that HAART is effective in preventing their occurrence only in the cases where $\mathrm{CD} 4^{+}$cell counts are restored to at least 500 cells per $\mathrm{mm}^{3}$ [45]. Indeed, circulating $\mathrm{CD}^{+}$cell count is the most predictive factor for all malignancies apart from anal cancer [46], which is better predicted by the duration of immunodeficiency.

Because the first reports between the association of HIV infection and neoplasia were published, there has been a dramatic change in the incidence and epidemiology of AIDS-related malignancies. KS, 
NHL and cervical cancer are classified by the Centers for Disease Control and Prevention as AIDS-defining malignancies. However, as the development and use of HAART continues, especially protease inhibitors, there has been a steady increase in non-AIDS-defining malignancies, such as Hodgkin's lymphoma (HL), lung cancer, hepatocellular cancer and anal cancer - and a decline in AIDSdefining neoplasias. These emerging malignancies present a new challenge in the care of patients with HIV infection, and require optimal treatment protocols that take into consideration the interaction between HAART and systemic chemotherapy [47].

\section{Anal cancer}

The incidence of anal cancer is progressively increasing among HIV-infected patients undergoing HAART [48]. The treatment of anal cancer relies upon traditional chemo- and radio-therapeutic approaches, which are associated with high morbidity and limited effectiveness in patients with high-grade disease. The role of mTOR in this setting has recently been investigated by Stelzer and colleagues [49] in preclinical models of murine and human anal cancer. The first model was based on the use of the HPV16 transgenic mouse, in which expression of the E6 and E7 oncogenes in the epithelium of the anus, along with the topical application of the carcinogen DMBA, causes the formation of a progressive neoplastic disease that leads to anal carcinoma. The second model comprises HPV16-positive human anal cancer xenografts passaged subcutaneously in immunodeficient SCID and nude mice. In both models, anal cancers were associated with activation of the mTOR pathway, and rapamycin significantly reduced their growth rates. In the transgenic mouse model the prophylactic administration of rapamycin also significantly reduced the incidence of the overall onset of tumours, which include benign and malignant lesions.

\section{Kaposi's sarcoma}

In the HAART era, KS remains the second most frequent tumour in HIV-infected patients worldwide, and the most common cancer in Sub-Saharan Africa. Patients with KS in Sub-Saharan Africa have high tumour burdens and rapid disease progression with a life expectancy $<6$ months. KS has a variable clinical course ranging from extremely indolent forms, requiring no or minimal therapy, to rapidly progressive disease. Several different therapeutic options are available but the optimal therapy is still unclear. HAART, including protease inhibitors, could represent the first treatment step for slowing progressive disease; chemotherapy plus HAART is indicated for visceral and/or rapidly progressive disease, whereas maintenance HAART after systemic chemotherapy could be an effective anti-KS measure. The angiogenic nature of KS makes it particularly suitable for therapies based on targeted agents such as metalloproteinase inhibitors, angiogenesis inhibitors and tyrosine kinase inhibitors [50].

The identification of the KS-associated herpes virus (KSHV or HHV8) as the viral etiologic agent of KS has prompted renewed interest in the molecular pathogenesis of this disease. Evidence now points to a single KSHV gene, $v G P C R$, as being essential for KS development, and recent work has identified the Akt/TSC/mTOR signalling cascade as a crucial pathway in vGPCR sarcomagenesis. Indeed, pharmacological inhibition of mTOR with rapamycin has shown promising results in preventing $v G P C R$-induced tumourigenesis in an animal model (see Ref. [51] for a review). These observations are further validated by coincidental reports demonstrating the efficacy of rapamycin as an immunosuppressive and antitumoural solution for post-transplant KS patients [52].

Although confirmation of these observations in controlled clinical trials is needed, these data seem to point to an important role for mTOR in the pathogenesis of KS and suggest that mTOR inhibitors could represent a novel therapeutic option for the treatment of this condition in HIV-infected individuals.

\section{Lymphomas}

Lymphomas comprise a large proportion of the malignancies that affect the HIV-infected population in developed countries. In particular, NHL represents the most common tumour in HIVinfected patients and represents an AIDS-defining illness since the establishment of HAART therapy [53].

The incidence of AIDS-related lymphoma (ARL) has been found to be 200-fold higher than the incidence of lymphoma in uninfected individuals. ARLs are often associated to herpes virus- $\gamma$, EBV or HHV-8 [43]. It is believed that B-cell proliferation stimulated by chronic antigenaemia can result in polyclonal and eventually monoclonal lymphoproliferation [43]. Standard treatment for HIV lymphomas includes immune reconstitution using HAART, anti-CD20 monoclonal antibodies, radioimmunotherapy, stem cell transplantation, cytokine therapy and vaccination. Because the prognosis for HIV-associated NHL nears that of the general population, these approaches are of increasing importance. However, treatment of HIV-associated lymphomas could be hindered by the difficulty of delivering full-dose chemotherapy to patients with compromised bone marrow or those suffering from infectious diseases. This might explain the lower responses and survival rates in HIV-associated lymphomas as compared with those of HIVseronegative patients [54]. Novel therapeutic options for HIVassociated lymphomas are therefore warranted.

El-Salem and colleagues [55] showed that the mTOR signalling pathway is activated in all patients with ARLs, regardless of their histological classification. In particular, they found that mTOR is activated in germinal centre cells and in the interfollicular areas, but not in the resting lymphocytes of the benign HIV-associated lymphadenopathy. Along with the reported occurrence of mTOR activation in non-HIV-associated lymphomas [56], these data suggest that the use of mTOR inhibitors could have potential therapeutic effects in HIV-associated and non-HIV-associated lymphomas. Although large studies to support the potential use of mTOR inhibitors against HIV-associated lymphomas are not available, it is interesting to observe that post-transplant lymphoproliferative disorder (PTLD)-type cell lines are highly sensitive to mTOR inhibition [57] and that substitution of immunosuppressive therapies based on inhibitors of calcineurin with mTORinhibitor-based therapies in organ-transplanted patients reduced the occurrence of PTLD lesions [58].

PEL constitutes a subset of NHL and PEL incidence is highly increased in HIV-infected patients. KS-associated herpes virus is the causative agent of PEL. PEL has a poor prognosis with reported median survival times of $<6$ months. PEL displays activated PI3K, Akt and mTOR. Although single modulation of the mTOR pathway inhibited PEL proliferation, it has been reported that dual inhibition of PI3K and mTOR was significantly more efficacious in culture and in a PEL xenograft tumour model [59]. These data 


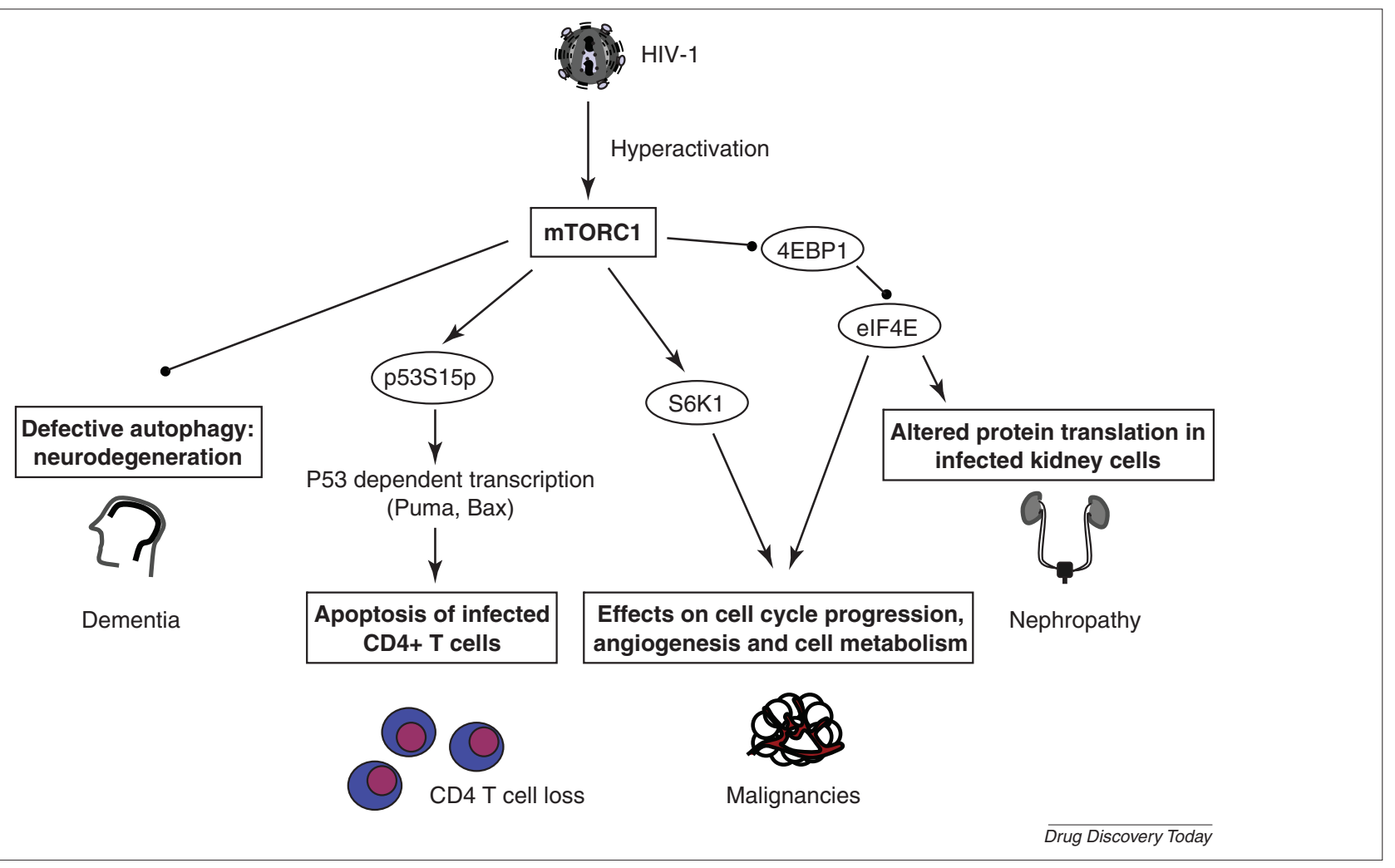

FIGURE 2

mTOR involvement in HIV-associated diseases. During HIV infection, defects in autophagy are involved in neurodegenerative processes that arise from glia. The decreased neuronal autophagy can sensitize cells to proapoptotic and other damaging mechanisms, leading to neuronal dysfunction and death. mTOR hyperactivation also seems to play a pathogenetic part in the dysregulated apoptosis of CD4 ${ }^{+} \mathrm{T}$ cells in HIV-1 patients through gp120-mediated activation of the mTOR-p53 axis. Finally, increased phosphorylation levels of S6K1 and 4EBP1 that occur upon HIV infection could also alter renal homeostasis causing nephropathy, and might be involved in cell growth and metabolism, which could lead to tumour development.

provide important preclinical proof-of-concept on the suitability of targeting the PI3K/mTOR axis with specific inhibitors for the treatment of PEL in HIV-infected patients [59].

Mantle-cell lymphoma (MCL), a well-defined subtype of B-cell non-Hodgkin's lymphoma (B-NHL), accounts for $\sim 6 \%$ of all lymphoid neoplasms, and has a median survival of 3-4 years. Recent studies show that most cases of MCL exhibit constitutive activation of PI3K/Akt/mTOR pathways, which promotes tumour proliferation and survival. In agreement with this, relapsed or refractory MCL patients in a Phase III clinical study have shown a better response to treatment with the specific mTOR inhibitor temsirolimus than the investigator's choice therapy [60]. These data warrant the use of MTOR inhibitors in HIV-associated MCL.

\section{Concluding remarks}

The data discussed indicate that hyperactivation of mTOR occurs in different pathologies associated with HIV infection such as nephropathy and AIDS-associated and non-AIDS-associated malignancies. Hyperactivation of mTOR could also be responsible for defective autophagy that can contribute to the pathogenesis of neurocognitive disorders during HIV infection (Fig. 2). Preclinical evidence indicates that selective inhibitors of mTOR such as rapamycin could represent a novel therapeutic approach for the treatment of these pathologies. HIV-infected patients could additionally benefit from the use of rapamycin because of its nonmTOR-mediated capacity to inhibit HIV replication [12]. Although, the use of an immunosuppressive drug such as rapamycin might raise the concern of increasing the immunodeficiency of HIV-infected patients, the fact that mTOR inhibitors have already proven effective in preventing some lymphoproliferative disorders in transplant patients, who are also immunocompromised [52,58], suggests that the direct effects of mTOR inhibitors could outweigh their immunosuppressive benefits in the clinical setting.

These data indicate that the potential use of mTOR inhibitors, either alone or in association with HAART, warrants that they are studied in Phase II proof-of-concept trials designed to determine whether the inhibition of mTOR ameliorates the natural course of the infection and reduces the risk of developing HIV-associated disorders.

\footnotetext{
References

1 Wullschleger, S. et al. (2006) TOR signaling in growth and metabolism. Cell 124 471-484

2 Zoncu, R. et al. (2011) mTOR: from growth signal integration to cancer, diabetes and ageing. Nat. Rev. Mol. Cell Biol. 12, 21-35
}

3 Sengupta, S. et al. (2010) Regulation of the mTOR complex 1 pathway by nutrients, growth factors, and stress. Mol. Cell 310-322

4 Düvel, K. (2010) Activation of a metabolic gene regulatory network downstream of mTOR complex 1. Mol. Cell 39, 171-183 
5 Sarbassov, D.D. et al. (2004) Rictor, a novel binding partner of mTOR, defines a rapamycin-insensitive and raptor-independent pathway that regulates the cytoskeleton. Curr. Biol. 14, 1296-1302

6 Vézina, C. et al. (1975) Rapamycin (AY-22,989), a new antifungal antibiotic. I. Taxonomy of the producing streptomycete and isolation of the active principle. $J$. Antibiot. (Tokyo) 28, 721-726

7 Hartford, C.M. and Ratain, M.J. (2007) Rapamycin: something old, something new, sometimes borrowed and now renewed. Clin. Pharmacol. Ther. 82 , 381-388

8 Malagelada, C. et al. (2010) Rapamycin protects against neuron death in in vitro and in vivo models of Parkinson's disease. J. Neurosci. 30, 1166-1175

9 Ehninger, D. and Silva, A.J. (2011) Rapamycin for treating Tuberous sclerosis and Autism spectrum disorders. Trends Mol. Med. 17, 78-87

10 Easton, J.B. and Houghton, P.J. (2006) mTOR and cancer therapy. Oncogene 25, 6436-6446

11 Sarbassov, D.D. et al. (2005) Growing roles for the mTOR pathway. Curr. Opin. Cell Biol. 17, 596-603

12 Donia, M. et al. (2010) Potential use of rapamycin in HIV infection. Br. J. Clin. Pharmacol. 70, 784-793

13 Nicoletti, F. et al. (2009) Inhibition of human immunodeficiency virus (HIV-1) infection in human peripheral blood leucocytes-SCID reconstituted mice by rapamycin. Clin. Exp. Immunol. 155, 28-34

14 Di Benedetto, F. (2010) First report on a series of HIV patients undergoing rapamycin monotherapy after liver transplantation. Transplantation 89, 733-738

15 Williams, K.C. and Burdo, T.H. (2009) HIV and SIV infection: the role of cellular restriction and immune responses in viral replication and pathogenesis. APMIS 117 , 400-412

$16 \mathrm{Wu}$, Y. and Yoder, A. (2009) Chemokine coreceptor signaling in HIV-1 infection and pathogenesis. PLoS Pathog. 5, e1000520

17 Cadogan, M. and Dalgleish, A.G. (2008) HIV immunopathogenesis and strategies for intervention. Lancet Infect. Dis. 8, 675-684

18 Mogensen, T.H. (2010) Innate immune recognition and activation during HIV infection. Retrovirology 7, 54

19 Grossman, Z. et al. (2006) Pathogenesis of HIV infection: what the virus spares is as important as what it destroys. Nat. Med. 12, 289-295

20 Hammer, S.M. et al. (2008) Antiretroviral treatment of adult HIV infection: recommendations of the International AIDS Society-USA panel. JAMA 300, $555-570$

21 Palella, F.J., Jr et al. (1998) Declining morbidity and mortality among patients with advanced human immunodeficiency virus infection. HIV Outpatient Study Investigators. N. Engl. J. Med. 338, 853-860

22 Baker, J.V. et al. (2008) Poor initial CD4 ${ }^{+}$recovery with antiretroviral therapy prolongs immune depletion and increases risk for AIDS and non-AIDS diseases. $J$. Acquir. Immune Defic. Syndr. 48, 541-546

23 Lau, B. et al. (2007) Risk of non-AIDS-related mortality may exceed risk of AIDSrelated mortality among individuals enrolling into care with $\mathrm{CD} 4^{+}$counts greater than 200 cells/mm3. J. Acquir. Immune Defic. Syndr. 44, 179-187

24 Perfettini, J.L. (2005) Mechanisms of apoptosis induction by the HIV-1 envelope. Cell Death Differ. 12 (Suppl. 1), 916-923

25 Castedo, M. et al. (2001) Human immunodeficiency virus 1 envelope glycoprotein complex-induced apoptosis involves mammalian target of rapamycin/FKBP12rapamycin-associated protein-mediated p53 phosphorylation. J. Exp. Med. 194, $1097-1110$

26 Espert, L. et al. (2006) Autophagy is involved in T cell death after binding of HIV-1 envelope proteins to CXCR4. J. Clin. Invest. 116, 2161-2172

27 Zhou, D. and Spector, S.A. (2008) Human immunodeficiency virus type-1 infection inhibits autophagy. AIDS 22, 695-699

28 Spector, S.A. and Zhou, D. (2008) Autophagy: an overlooked mechanism of HIV-1 pathogenesis and neuroAIDS? Autophagy 4, 704-706

29 Kedzierska, K. et al. (2003) Defective phagocytosis by human monocyte/ macrophages following HIV-1 infection: underlying mechanisms and modulation by adjunctive cytokine therapy. J. Clin. Virol. 26, 247-263

30 Van Grol, J. (2010) HIV-1 inhibits autophagy in bystander macrophage/monocytic cells through Src-Akt and STAT3. PLOS ONE 5, e11733

31 Jagannath, C. et al. (2009) Autophagy enhances the efficacy of BCG vaccine by increasing peptide presentation in mouse dendritic cells. Nat. Med. 15, 267-276
32 Blanchet, F.P. et al. (2010) Human immunodeficiency virus-1 inhibition of immunoamphisomes in dendritic cells impairs early innate and adaptive immune responses. Immunity 32, 654-669

33 McArthur, J.C. et al. (2010) Human immunodeficiency virus-associated neurocognitive disorders: mind the gap. Ann. Neurol. 67, 699-714

34 Simioni, S. et al. (2010) Cognitive dysfunction in HIV patients despite long-standing suppression of viremia. AIDS 24, 1243-1250

35 Kibayashi, K. et al. (1996) Neuropathology of human immunodeficiency virus infection at different disease stages. Hum. Pathol. 27, 637-642

36 Alirezaei, M. et al. (2008) Decreased neuronal autophagy in HIV dementia: a mechanism of indirect neurotoxicity. Autophagy 4, 963-966

37 Alirezaei, M. et al. (2008) Disruption of neuronal autophagy by infected microglia results in neurodegeneration. PLOS ONE 3, e2906

38 Nardacci, R. et al. (2005) Characterization of cell death pathways in human immunodeficiency virus-associated encephalitis. Am. J. Pathol. 167, 695-704

39 Atta, M.G. (2010) Diagnosis and natural history of HIV-associated nephropathy. Adv. Chronic Kidney Dis. 17, 52-58

40 Kaufman, L. et al. (2010) The pathogenesis of HIV-associated nephropathy. Adv. Chronic Kidney Dis. 17, 36-43

41 Wyatt, C.M. et al. (2008) HIV-associated nephropathy: clinical presentation, pathology, and epidemiology in the era of antiretroviral therapy. Semin. Nephrol. 28, 513-522

42 Kumar, D. et al. (2010) HIV-associated nephropathy: role of mammalian target of rapamycin pathway. Am. J. Pathol. 177, 813-821

43 Wood, C. and Harrington, W., Jr (2005) AIDS and associated malignancies. Cell Res. $15,947-952$

44 Clifford, G.M. and Franceschi, S. (2009) Cancer risk in HIV-infected persons: influence of CD4(+) count. Fut. Oncol. 5, 669-678

45 Barbaro, G. and Barbarini, G. (2007) HIV infection and cancer in the era of highly active antiretroviral therapy. Oncol. Rep. 17, 1121-1126

46 Guiguet, M. et al. (2009) Clinical Epidemiology Group of the FHDH-ANRS CO4 cohort. Effect of immunodeficiency, HIV viral load, and antiretroviral therapy on the risk of individual malignancies (FHDH-ANRS CO4): a prospective cohort study. Lancet Oncol. 10, 1152-1159

47 Cáceres, W. et al. (2010) AIDS-related malignancies: revisited. P. R. Health Sci. J. 29, $70-75$

48 Silverberg, M.J. and Abrams, D.I. (2009) Do antiretrovirals reduce the risk of nonAIDS-defining malignancies? Curr. Opin. HIV AIDS 4, 42-51

49 Stelzer, M.K. (2010) Rapamycin inhibits anal carcinogenesis in two preclinical animal models. Cancer Prev. Res. (Phila.) 3, 1542-1551

50 Martellotta, F. et al. (2009) AIDS-related Kaposi's sarcoma: state of the art and therapeutic strategies. Curr. HIV Res. 7, 634-638

51 Montaner, S. (2007) Akt/TSC/mTOR activation by the KSHV G protein-coupled receptor: emerging insights into the molecular oncogenesis and treatment of Kaposi's sarcoma. Cell Cycle 6, 438-443

52 Stallone, G. (2005) Sirolimus for Kaposi's sarcoma in renal-transplant recipients. $N$. Engl. J. Med. 352, 1317-1323

53 Carbone, A. (2002) AIDS-related non-Hodgkin's lymphomas: from pathology and molecular pathogenesis to treatment. Pathology 33, 392-404

54 Yarchoan, R. (2005) Therapy insight: AIDS-related malignancies - the influence of antiviral therapy on pathogenesis and management. Nat. Clin. Pract. Oncol. 2, 406-415

55 El-Salem, M. et al. (2009) Activation of mTORC1 signaling pathway in AIDS-related lymphomas. Am. J. Pathol. 175, 817-824

56 Leseux, L. et al. (2006) Syk-dependent mTOR activation in follicular lymphoma cells. Blood 108, 4156-4162

57 Majewski, M. et al. (2003) Immunosuppressive signal transduction inhibitor RAD suppresses growth of cells derived from post-transplant lymphoproliferative disorder at allograft protecting doses. Transplantation 75, 1710-1717

58 Boratynska, M. and Smolska, D. (2008) Inhibition of mTOR by sirolimus induces remission of post-transplant lymphoproliferative disorders. Transpl. Int. 21, 605-608

59 Bhatt, A.P. et al. (2010) Dual inhibition of PI3K and mTOR inhibits autocrine and paracrine proliferative loops in PI3K/Akt/mTOR-addicted lymphomas. Blood 115, 4455-4463

60 Hess, G. et al. (2009) Phase III study to evaluate temsirolimus compared with investigator's choice therapy for the treatment of relapsed or refractory mantle cell lymphoma. J. Clin. Oncol. 27, 3822-3829 